\title{
EFFECT OF REPEATED DEEP-FAT FRYING OF FRENCH FRIES IN SUNFLOWER AND PALM OILS ON THE ACRYLAMIDE CONTENT
}

\author{
Adriana Laura Mihai ${ }^{1}$, Mioara Negoiță ${ }^{1 *}$, Gabriela-Andreea Horneț ${ }^{1}$, Alina Cristina Adascăluluii ${ }^{1}$ \\ ${ }^{1}$ National Research \& Development Institute for Food Bioresources, IBA Bucharest, Chromatography Department, 6
} Dinu Vintilă Street, District 2, 021102, Bucharest, Romania

\begin{abstract}
The study aimed to investigate the influence of repeated frying processes of potatoes in a fryer on the acrylamide content of French fries. For deep-frying, potatoes of the Asinaria variety, sunflower and palm oils were used. For each oil type, five frying cycles at $170^{\circ} \mathrm{C}$, for $6 \mathrm{~min}$ were performed and the acrylamide content of the final product was analyzed using the solid phase extraction (SPE) technique and the GC-MS/MS method. The acrylamide content increased in the case of sunflower oil in the first three frying cycles, while for palm oil it increased in the fourth frying cycles, then a decrease was recorded. The mean acrylamide content of French fries samples fried in sunflower oil $(1477.07 \mu \mathrm{g} / \mathrm{kg})$ was higher than the one of French fries fried in palm oil (1258.95 $\mu \mathrm{g} / \mathrm{kg})$. For all samples analyzed, the acrylamide content exceeded the benchmark level of $500 \mu \mathrm{g} / \mathrm{kg}$ settled by the EU 2017/2158. The acrylamide content of French fries was correlated with the color parameter $L^{*}$.
\end{abstract}

Keywords: acrylamide, French fries, frying oil, palm oil, sunflower oil

\section{INTRODUCTION}

It is well known that at temperatures higher than $120^{\circ} \mathrm{C}$, the chemical process contaminant acrylamide is formed as a result of the Maillard reaction, which occurs between asparagine and reducing sugars. Besides the Maillard pathway of acrylamide formation, another way to form this compound is the acrolein way, by lipid oxidation, which involves glycerol degradation, followed by acrolein oxidation to acrylic acid which reacts with ammonia like this resulting acrylamide (Becalski et al., 2003).

French fries are prone to form acrylamide as potatoes contain high amounts of asparagine and reducing sugars and as this product is exposed to oil oxidation while frying. French fries are products that are consumed regularly, being served at restaurants, canteens, fast foods and also home-made, being one of the most important food groups that contribute to acrylamide exposure especially for adults and children (EFSA, 2015). The acrylamide content of French fries is influenced by various factors such as: the potato cultivator, fertilizer used, storage conditions, pretreatments applied, cooking conditions (temperature, time), amount of precursors found in the potatoes, also the frying oil used (Yang et al., 2016).

French fries are exposed to frying, a cooking process in which the products are immersed in hot oils, and by which the properties of raw materials are changed. By the exposure of oil to frying, it 
degrades and changes its physical-chemical properties as a result of the generation of surface-active substances. Surface tension is an important factor, which affects the oil uptake.

For potatoes frying, the most used vegetable oils are sunflower and palm oils. Palm oil at room temperature is semi-solid, while by heating it, the viscosity decreases with increasing the temperature $\left(40-200^{\circ} \mathrm{C}\right.$ ) (Sahasrabudhe et al., 2019). Kalogianni et al. (2011) showed that by repeatedly frying and heating of potatoes, palm oil viscosity suffered a greater increase compared to the olive oil as a result of heat and mass transfer changes during frying. Vegetable oils high in unsaturated fatty acids, as sunflower oil are more susceptible to oxidation and polymerization during frying comparing with oils with an equilibrated proportion of saturated and unsaturated fatty acids which are more suitable to frying (Abd Razak et al., 2021).

The effect of frying cycles on acrylamide formation was investigated in different studies (Santos et al., 2018; Kuek et al., 2020) as this culinary practice is used generally in fast-foods. By repeated frying, the physical-chemical properties of oil are changed by oxidation and oils are more susceptible to form acrylamide.

Due to the fact that acrylamide is considered a public health concern (EFSA, 2015), the European Commission established the regulation (EU) 2017/2158 which presents the mitigation measures and benchmark levels for the reduction of the presence of acrylamide in food. This regulation settled a benchmark level of $500 \mu \mathrm{g} / \mathrm{kg}$ for ready-to-eat French fries.

Therefore, the aim of this work was to study the influence of repeated deep-frying cycles of potatoes in sunflower and palm oils on the acrylamide level formed in French fries, and to establish a correlation between the acrylamide content and color parameters of the end-products.

\section{MATERIALS AND METHODS}

\section{Chemicals, reagents and standards}

Native acrylamide (min. 99\% purity) purchased from Restek (Benner Circle, Bellefonte, USA) and internal standard (IS) of labeled acrylamide $\left(1,2,3-{ }^{13} \mathrm{C}\right.$ labeled acrylamide, min. 99\% purity) obtained from Cambridge Isotope Laboratories (Andover, MA, USA) were used. All other chemicals, reagents and solvents were of analytical grade. The SPE cartridges used in this study were Isolute $^{\circledR}$ Multimode $(1000 \mathrm{mg}, 6 \mathrm{~mL})$ and Isolute ${ }^{\circledR} \mathrm{ENV}+(500 \mathrm{mg}, 6 \mathrm{~mL})$, which were supplied by Biotage (Uppsala, Sweden).

\section{Samples}

Potatoes of Asinaria variety obtained from the National Research and Development Institute for Potato and Sugar Beet Brașov, Romania were used. Potatoes were harvested in 2020 and experiments were realized in February 2021.

Vegetable oils of sunflower and palm coming from the same batch were purchased from a local supermarket from Bucharest, Romania.

\section{Sample preparation}

Potatoes were peeled and cut at the dimension of $9 \mathrm{~mm} \times 9 \mathrm{~mm}$ with a length between 60 and 80 $\mathrm{mm}$. After this step, potatoes were rinsed in tap water, and the excess water from the potato surface was removed using absorbent paper. For this experiment, palm and sunflower oils were weighted and transferred in the fryer up to the low level.

Frying was realized in a temperature-controlled fryer with two unites of customized 4 L capacity stainless (Hendi Blue Line), in which oils were gradually heated at $170^{\circ} \mathrm{C}$, and maintained at this 
temperature for $30 \mathrm{~min}$ before frying the potatoes. For each frying cycle a $1 / 4$ potato/oil ratio $(\mathrm{w} / \mathrm{w})$ was used, which was chosen as the potatoes to be deep fried. Potatoes were fried at $170^{\circ} \mathrm{C}$, for 6 min. The frying cycle was repeated each $15 \mathrm{~min}$. At the end of the frying cycle, the frying basket with the sample was taken out and shaken. French fries were let to cool on a plate and after this it were ground and homogenized in a laboratory mixer (Büchi, Labortechnik AG, Germany) and then it were transferred in a $50 \mathrm{~mL}$ centrifuge tube and kept at $-20^{\circ} \mathrm{C}$ before analyses. About $15 \mathrm{~g}$ of oil was collected after each frying cycle in order to analyze the color, quantity which was replaced with fresh oil to maintain the oil level. The oil was kept at room temperature in amber vials until color parameters were determined.

\section{Acrylamide determination by GC-MS/MS}

Acrylamide extraction and determination were performed on the basis of the optimized method previously described by Negoiță et al. (2020). Briefly, acrylamide extraction was realized by the SPE method and for the acrylamide quantification a gas chromatograph in tandem with mass spectrometer (GC-MS/MS) was used.

For acrylamide quantification two calibration curves in the range of $0.05-3 \mathrm{mg} / \mathrm{L}$ were used, which had correlation coefficients higher than 0.999 . The method recovery was between 85.64 and 109.22\%. The limit of detection (LOD) and limit of quantification (LOQ) were 10.29 and 30.87 $\mu \mathrm{g} / \mathrm{kg}$, respectively.

\section{Color determination}

Color measurements were performed using a Konica Minolta spectrophotometer (Universal Software V4.01 Miniscan XE Plus). The spectrophotometer was calibrated using white and black plates provided by the manufacturer. Color measurements for French fries and oils were realized by using illuminate D65 with an angle of view of $10^{\circ}$. For French fries samples, 10 measurements of the color parameters $L^{*}$ (lightness), $a^{*}$ (negative values indicate green and positive values indicate red), and $b^{*}$ (negative values indicate blue and positive values indicate yellow) were performed on different points of the grounded samples, while for oils 2 measurements were performed.

\section{Peroxide value}

The peroxide index of sunflower and palm oils after each frying cycle was performed according to SR EN ISO 3960:2017. It is expressed in milliequivalents of peroxide per $1 \mathrm{~kg}$ of product.

\section{Statistical analysis}

For acrylamide determination, French fries samples were analyzed in duplicate, and results were expressed as mean \pm standard deviation. In the case of color parameters, 10 measurements were realized for French fries and 2 measurements for oils, and results were expressed as mean \pm standard deviation.

\section{RESULTS AND DISCUSSIONS Acrylamide content}

Often, in different fast-foods and restaurants, the potatoes are fried repeatedly in the same oil. Exposed to high temperatures, the oil suffers a series of physical-chemical reactions, which have direct consequences on the taste, smell, texture or chemical composition of potatoes. During frying, the chemical contaminant acrylamide can be formed. Besides acrylamide, during frying the trans 
fatty acids content of vegetable oil increase, as a result of oil thermal degradation (Santos et al., 2018).

For this study, the influence of five frying cycles on the acrylamide concentrations of French fries was investigated. The frying process was realized in a fryer set to $170^{\circ} \mathrm{C}$ and potato strips were maintained for $6 \mathrm{~min}$ in the fryer in palm and sunflower oils. The frying temperature was chosen based on the recommendation from Commission Regulation (EU) 2017/2158 which specifies a frying temperature below $175^{\circ}$. The acrylamide content of the French fries samples is presented in figure 1.

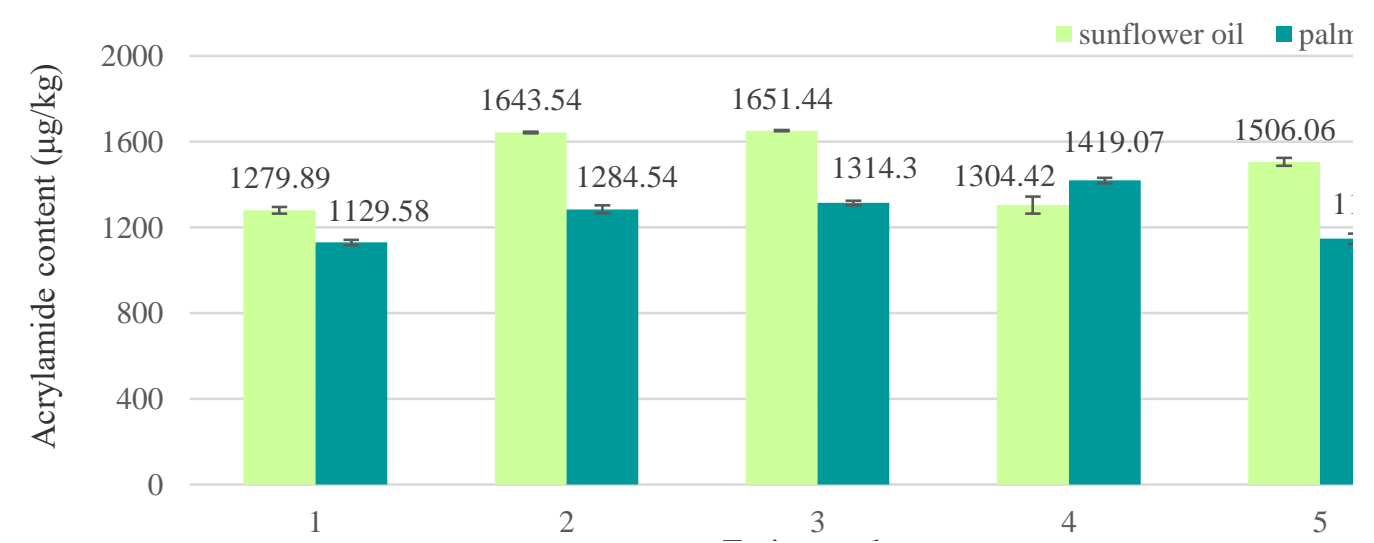

Figure 1. Acrylamide concentration $(\mu \mathrm{g} / \mathrm{kg})$ of French fries samples after frying at $170^{\circ} \mathrm{C}$ for 6 min

For all analyzed samples, the acrylamide content exceeded the benchmark level of $500 \mu \mathrm{g} / \mathrm{kg}$ established by the Commission Regulation (EU) 2017/2158.

In the study realized by Mesias et al. (2017) it was shown that by purchasing the same variety of potatoes in two consecutive months, the reducing sugar content increased for the potatoes purchased in the second month, and as a result, the acrylamide content was higher, ranging between 1932$2116 \mu \mathrm{g} / \mathrm{kg}$ compared with the ones purchased in the first month who had an acrylamide content ranging between 1513-1847 $\mu \mathrm{g} / \mathrm{kg}$. The high acrylamide content of French fries obtained in our study can be a result of the storage conditions, the quantity of reducing sugars increasing during storage.

The mean acrylamide content of potatoes fried in sunflower oil $(1477.07 \pm 178.64 \mu \mathrm{g} / \mathrm{kg})$ was higher than the one obtained when palm oil $(1258.95 \pm 121.01 \mu \mathrm{g} / \mathrm{kg})$ was used. In the study realized by Kuek et al. (2020) results were in accordance with the one obtained in our study, a lower level of acrylamide being found in French fries fried in palm olein $(452 \mu \mathrm{g} / \mathrm{kg})$, than in the ones fried in sunflower oil $(500 \mu \mathrm{g} / \mathrm{kg})$. It is known that palm oil contains a higher concentration of saturated fatty acids than sunflower oil (Mihai et al., 2019), which leads to a smaller quantity of oil absorbed by the food (Osório and de Lourdes Cardeal, 2011). Also, oils with a high degree of unsaturation tend to oxidate and polymerize faster during frying process compared to oils with a lower level of unsaturation (Abd Razak et al., 2021). Another factor that can influence the acrylamide content of French fries is the heat transfer coefficient of oils used for frying. Potatoes fried in oils with lower heat transfer coefficients form a lower level of acrylamide, while when a higher heat transfer coefficient develop more acrylamide in the end product (Zhang et al., 2015). 


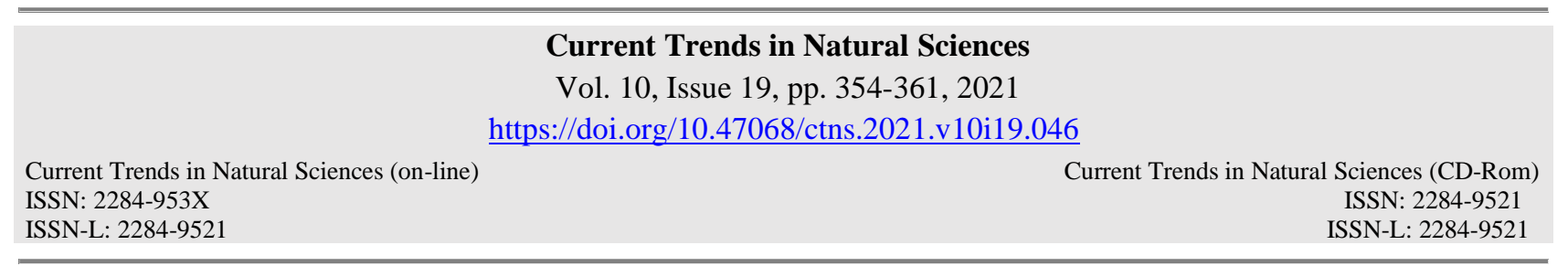

The flash point for sunflower oil at $210^{\circ} \mathrm{C}$ is $316^{\circ} \mathrm{C}$, while for palm oil it is $280^{\circ} \mathrm{C}$ (Gomna et al., 2019).

For both types of oil used, the lowest acrylamide content was obtained for French fries after the first frying cycle. However, our results are not in agreement with the one reported by Kuek et al. (2020) who found an acrylamide content higher for the first frying cycle of French fries prepared in red palm olein, sunflower and soybean oils.

Osório and de Lourdes Cardeal (2011) studied the influence of four frying cycles of French fries in sunflower, palm, soybean, corn and canola oils at $170^{\circ} \mathrm{C}$ for $12-15$ minutes on the acrolein content of the final products. In the case of potatoes fried in sunflower and palm oils, the acrolein content decreased after the $2^{\text {nd }}$ and $3^{\text {rd }}$ frying cycles, while a significant increase was observed at the $4^{\text {th }}$ frying cycle. Results are in agreement with the ones obtained in our study, acrolein being consumed to form acrylamide in the first three frying cycles, while in the fourth one, acrolein increased, being correlated with a smaller amount of acrylamide. In the study realized by Santos et al. (2018), it was shown that the acrylamide content of French fries fried at $175^{\circ} \mathrm{C}$ in extra-virgin olive oil, peanut and canola oils was not affected by the oil used but by frying hours, in special for olive and canola oils. In contrast, Abd Razak et al. (2021) concluded that the oil type influences the acrylamide content of French fries, increasing after $80 \mathrm{~h}$ of frying, the highest content being found for canola oil, followed by soybean oil, sunflower oil and palm olein.

\section{Peroxide index of oils used}

Repeated usage of oil when frying potatoes leads to its deterioration during frying, through changes in the chemical composition, especially oxidizing substances. Figure 2 shows that as the number of frying cycle increased, the oil quality degraded, like this resulting the increase of the peroxide index.

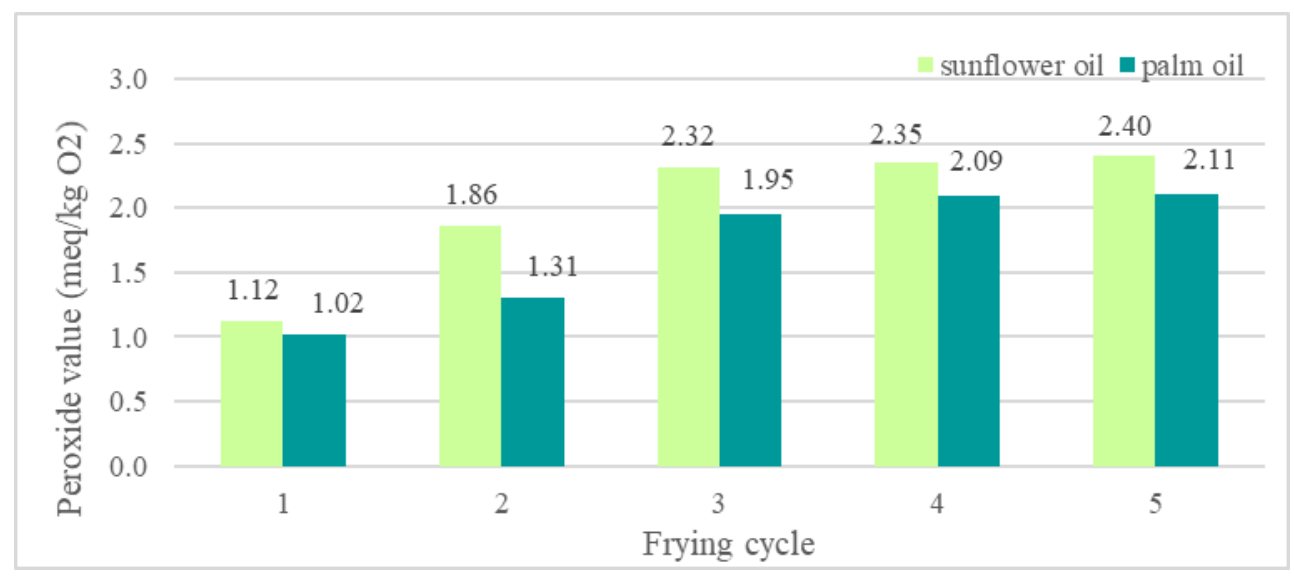

Figure 2. Peroxide value of vegetable oils used for the frying cycles

For both oils analyzed, the peroxide index increased from the first frying cycle to the fifth frying cycle, which indicates that oil oxidation was produced. In the case of sunflower oil, the peroxide index had higher values compared to palm oil. Similar results were obtained by Kuek et al. (2020) 


\section{Current Trends in Natural Sciences}

Vol. 10, Issue 19, pp. 354-361, 2021

https://doi.org/10.47068/ctns.2021.v10i19.046

Current Trends in Natural Sciences (on-line)

who obtained lower values of peroxide index when palm oil was used for intermittent frying of French fries compared to sunflower oil.

\section{Color parameters}

\section{Oils color parameters}

Color parameters of fresh palm and sunflower oils and of the ones collected after the five frying cycles were performed using the spectrophotometer and results are presented in table 1. Oil color is influenced by the oxidation process. The highest lightness ( $L^{*}$ parameter) was observed for fresh oils, before being subjected to frying. However, no significant differences were observed after frying the oil. For the color parameter $a^{*}$, it was observed that it had a negative value which decreased in the fifth frying cycles, meaning that the color is in the green spectrum. The highest $a^{*}$ value was obtained for sunflower oil. In the case of color parameter $b^{*}$, the yellowness of both oils used it was noticed an increase compared to the fresh oils. Palm oil presented a higher value of color parameter $b^{*}$ which indicate that the oil became yellower after the frying treatments.

Table 1. Color parameters of fresh vegetables oils and after the frying cycles at $170^{\circ} \mathrm{C}$ for 6 min

\begin{tabular}{|l|l|l|l|l|l|l|l|}
\hline \multirow{2}{*}{$\begin{array}{l}\text { Color } \\
\text { parameters }\end{array}$} & $\begin{array}{l}\text { Vegetable oils } \\
\text { used for } \\
\end{array}$ & Frying cycle & Fresh & $1^{\text {st }}$ & $2^{\text {nd }}$ & $3^{\text {rd }}$ & $4^{\text {th }}$ \\
\hline \multirow{2}{*}{$L^{*}$} & Palm oil & $94.90 \pm 0.01$ & $93.55 \pm 0.06$ & $93.67 \pm 0.01$ & $93.73 \pm 0.03$ & $93.74 \pm 0.03$ & $93.65 \pm 0.01$ \\
\cline { 2 - 8 } & Sunflower oil & $95.48 \pm 0.01$ & $94.47 \pm 0.00$ & $95.45 \pm 0.00$ & $95.47 \pm 0.01$ & $95.42 \pm 0.00$ & $95.41 \pm 0.00$ \\
\hline \multirow{2}{*}{$a^{*}$} & Palm oil & $-1.12 \pm 0.01$ & $-1.42 \pm 0.01$ & $-1.47 \pm 0.00$ & $-1.57 \pm 0.01$ & $-1.6 \pm 0.01$ & $-1.68 \pm 0.01$ \\
\cline { 2 - 8 } & Sunflower oil & $-0.44 \pm 0.01$ & $-0.53 \pm 0.01$ & $-0.55 \pm 0.00$ & $-0.57 \pm 0.00$ & $-0.59 \pm 0.00$ & $-0.61 \pm 0.00$ \\
\hline \multirow{2}{*}{$b^{*}$} & Palm oil & $5.20 \pm 0.00$ & $8.01 \pm 0.02$ & $7.99 \pm 0.02$ & $7.96 \pm 0.02$ & $8.26 \pm 0.01$ & $8.43 \pm 0.01$ \\
\cline { 2 - 8 } & Sunflower oil & $1.48 \pm 0.00$ & $1.70 \pm 0.01$ & $1.76 \pm 0.00$ & $1.80 \pm 0.00$ & $1.85 \pm 0.01$ & $1.91 \pm 0.00$ \\
\hline
\end{tabular}

\section{French fries color parameters}

Color is an important factor which shows the acrylamide content of food products. It can be used as an indicator to test the quantity of reducing sugars from potatoes prior to use. It is recommended that potatoes to be cook until a golden yellow color for the finished product $(\mathrm{EC}, 2017)$ in order to form a lower level of acrylamide.
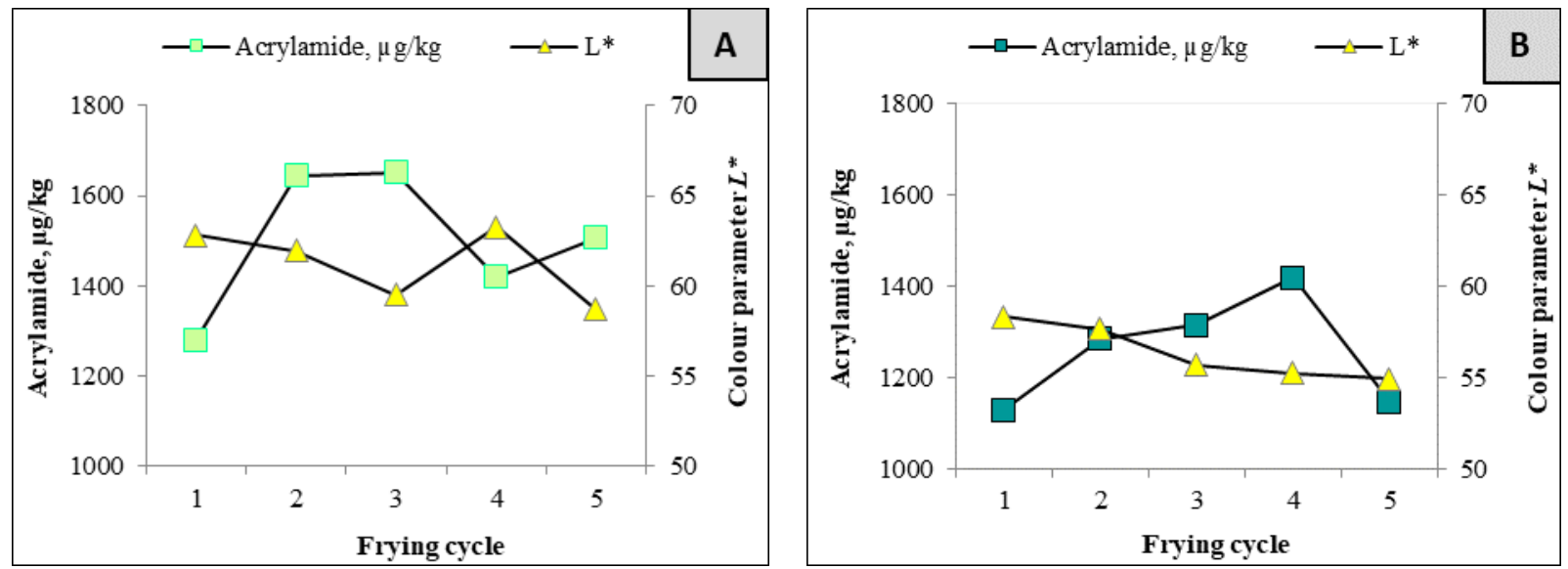

Figure 3. Variation of acrylamide content and color parameter $L^{*}$ of French fries fried in sunflower oil (A) and palm oil (B) 


\begin{tabular}{|c|c|}
\hline & $\begin{array}{r}46 \\
\end{array}$ \\
\hline $\begin{array}{l}\text { Current Trends in Natural Sciences (on-line) } \\
\text { ISSN: 2284-953X } \\
\text { ISSN-L: 2284-9521 }\end{array}$ & $\begin{array}{r}\text { Current Trends in Natural Sciences (CD-Rom) } \\
\text { ISSN: 2284-9521 } \\
\text { ISSN-L: 2284-9521 }\end{array}$ \\
\hline
\end{tabular}

Figure 3 show the variation of acrylamide level and color parameter $L^{*}$ of French fries after the repeated deep-fat frying in the same oil for five times, at $170^{\circ} \mathrm{C}$, for 6 minutes, while figures 4 and 5 shows images of French fries samples after the frying cycles.

It can be noted that French fries fried in sunflower oil had a lighter color than the ones fried in palm oil. In the case of color parameter $L^{*}$, it was recorded a decrease for all the frying cycle, French fries samples became darker after frying. For sunflower oil it was noted a decrease for the first three frying cycles, while in the fourth frying cycle it increased, and in the fifth cycle it decreased again. This variation was correlated with the acrylamide content which increased in the first three frying cycle, then it decreased, being followed by an increase in the last frying cycle. Also, when palm oil was used for frying, the color parameter $L^{*}$ of French fries was correlated with the acrylamide variation. No correlation was found between the acrylamide content and color parameters $a^{*}$ and $b^{*}$.
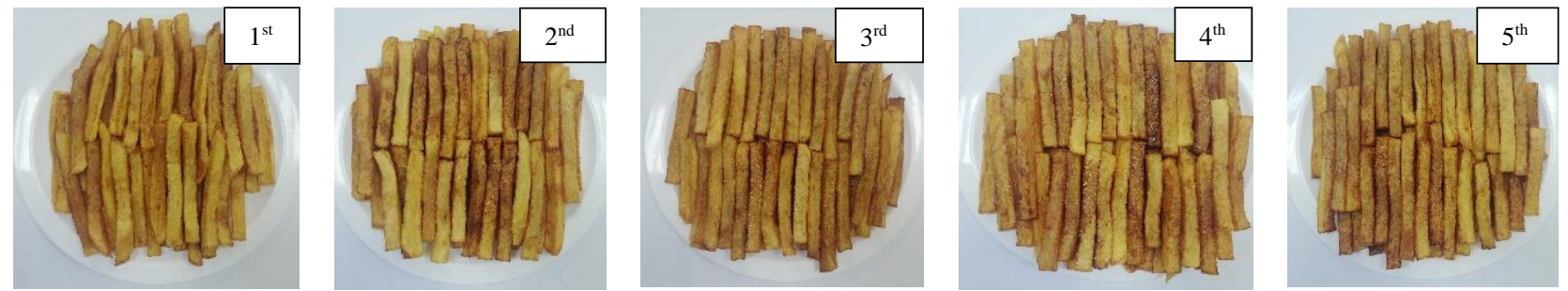

Figure 4. Images of French fries samples after the repeated frying cycles in palm oil at $170^{\circ} \mathrm{C}$ for 6 min
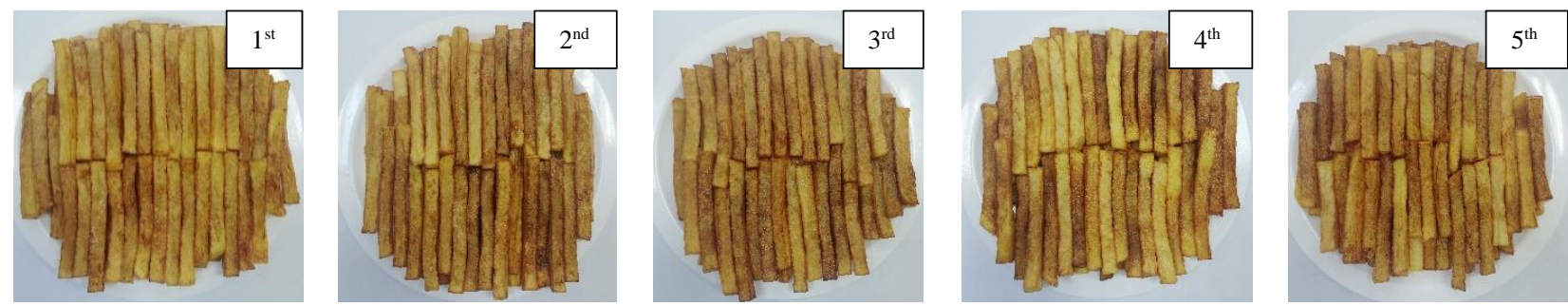

Figure 5. Images of French fries samples after the repeated frying cycles in sunflower oil at $170^{\circ} \mathrm{C}$ for 6 min

\section{CONCLUSIONS}

Results of this study showed that the frying oil and the repeated deep-fat frying of potatoes influence the acrylamide content in French fries. The acrylamide level of French fries fried in sunflower oil increased in the first three frying cycles from 1279.89 to $1651.44 \mu \mathrm{g} / \mathrm{kg}$, while when palm oil was used, it increased in the first four frying cycle from 1129.58 to $1419.07 \mu \mathrm{g} / \mathrm{kg}$

In general, the acrylamide content of French fries fried in palm oil was lower than when sunflower oil was used. This can be the result of the fatty acids composition of palm oil which is more stable to frying process than sunflower oil.

\section{ACKNOWLEDGEMENTS}

This study was achieved through Core Programme (PN 19 02), with the support of the Ministry of Research and Innovation (MCI), contract 22N/2019, project PN 19020301. 


\section{Current Trends in Natural Sciences}

Vol. 10, Issue 19, pp. 354-361, 2021

https://doi.org/10.47068/ctns.2021.v10i19.046

Current Trends in Natural Sciences (on-line)

\section{REFERENCES}

Abd Razak, R., Tarmizi, A.H.A., Kuntom, A., Sanny, M., Ismail, I.S. (2021). Intermittent frying effect on French fries in palm olein, sunflower, soybean and canola oils on quality indices, 3-monochloropropane-1,2-diol esters (3MCPDE), glycidyl esters (GE) and acrylamide contents. Food Control, 124, 107887.

Becalski, A., Lau, B.P.-Y., Lewis, D., Seaman, S.W. (2003). Acrylamide in food: occurrence, sources, and modeling. $J$ Agric Food Chem. 51(3), 802-808.

Commission Regulation 2017/2158 establishing mitigation measures and benchmark levels for the reduction of the presence of acrylamide in food. Official Journal of the European Union. 2017; L 304/24

European Food Safety Authority (EFSA). 2015. Scientific opinion on acrylamide in food. EFSA Panel on contaminants in the food chain (CONTAM). EFSA Journal. 13(6):4104

Gomna, A., N'Tsoukpoe, K.E., Le Pierrès, N. Coulibaly, Y. (2019). Review of vegetable oils behaviour at high temperature for solar plants: Stability, properties and current applications. Sol. Energy Mater Sol. Cells. 200, 109956.

Kalogianni, E.P., Karapantsios, T.D., Miller, R. (2011). Effect of repeated frying on the viscosity, density and dynamic interfacial tension of palm and olive oil. J Food Eng. 105, 169-179.

Kuek, S.L., Tarmizi, A.H.A., Razak, R.A., Jinap, S., Norliza, S., Sanny, M. (2020). Contribution of lipid towards acrylamide formation during intermittent frying of French fries. Food Control, 118, 107430.

Mesías, M, Holgado, F, Márquez-Ruiz, G, Morales, FJ. (2017). Impact of the characteristics of fresh potatoes available in-retail on exposure to acrylamide: Case study for French fries. Food Control, 73, 1407-1414.

Mihai, A.L., Negoiță, M., Belc, N. (2019). Evaluation of fatty acid profile of oils/fats by GC-MS through two quantification approaches. Rom Biotechnol Lett, 24(6), 973-985.

Negoiţă, M., Mihai, L.A., Horneț, G.A., Duţă, D.E. (2020). Development of SPE clean-up procedure for acrylamide determination from potato-based products by GC-MS/MS. DE GRUYTER, Open Agriculture. 5, 305-316.

Osório, V.M., de Lourdes Cardeal, Z. (2011). Determination of acrolein in French fries by solid-phase microextraction gas chromatography and mass spectrometry. J. Chromatogr. A, 1218, 3332-3336.

Sahasrabudhe, S.N., Staton, J.A., Farkas, B.E. (2019). Effect of frying oil degradation on surface tension and wettability. LWT - Food Sci Technol., 99, 519-524.

Santos, C.S.P., Molina-Garcia, L., Cunha, S.C., Casal, S. (2018). Fried potatoes: Impact of prolonged frying in monounsaturated oils. Food Chem, 243, 192-201.

SR EN ISO 3960:2017. Animal and vegetable fats and oils. Determination of the peroxide index. Determination with iodometric stopping point (visual)

Yang, Y., Achaerandio, I., Pujolà, M. (2016). Influence of the frying process and potato cultivar on acrylamide formation in French fries. Food Control, 62, 216-223.

Zhang, H., Cheng, L., Wang, L., Qian, H. (2015). Influence of deep-frying using various commercial oils on acrylamide formation in French fries. Food Addit Contam Part A, 32(7), 1083-1088. 